\title{
A hybrid approach for reliability analysis based on analytic hierarchy process and Bayesian network
}

\author{
Muhammad Zubair* \\ Department of Basic Sciences, University of Engineering and Technology Taxila, Taxila, Pakistan
}

Edited by:

Wuhua Duan, Tsinghua University, China

\section{Reviewed by:}

Minjun Peng, Harbin Engineering

University, China

Khalil Ur Rahman, Kyung Hee

University, South Korea

Sajjad Ahmed, Government College

Satellite Town, Pakistan

${ }^{*}$ Correspondence:

Muhammad Zubair, Department of Basic Sciences, University of

Engineering and Technology Taxila,

Taxila, Pakistan

e-mail:zubairheu@gmail.com
By using analytic hierarchy process (AHP) and Bayesian Network (BN) the present research signifies the technical and non-technical issues of nuclear accidents. The study exposed that the technical faults was one major reason of these accidents. Keep an eye on other point of view it becomes clearer that human behavior like dishonesty, insufficient training, and selfishness are also play a key role to cause these accidents. In this study, a hybrid approach for reliability analysis based on AHP and BN to increase nuclear power plant (NPP) safety has been developed. By using AHP, best alternative to improve safety, design, operation, and to allocate budget for all technical and non-technical factors related with nuclear safety has been investigated. We use a special structure of BN based on the method AHP. The graphs of the BN and the probabilities associated with nodes are designed to translate the knowledge of experts on the selection of best alternative. The results show that the improvement in regulatory authorities will decrease failure probabilities and increase safety and reliability in industrial area.

\section{Keywords: reliability, AHP, BN, nuclear energy, accident analysis}

\section{INTRODUCTION}

The advance technology has tendency to built nuclear power plants (NPPs) with large capacity and capital cost that makes system operating conditions more complex. As a result safety and reliability becomes more important. The probabilistic safety assessment (PSA) approach has potential to process historical, design, and operation data to generate results for decision making. For this purpose, different techniques have been used in PSA including fault tree analysis (FTA), reliability block diagram (RBD), and multi-attribute utility theory (MAUT) (NUREG-0492, 1981; Youngblood, 1998; Apostolakis and Lemon, 2005; Ahmed et al., 2011). In PSA, we use FTA and ETA to model failure rates, large FT and small ET is one way for modeling, large ET and small FT is another method to represents failure rates (Haasl et al., 1985). By using FT it becomes easy to model all possible ways that cause top event and probability of each event. These failure probabilities also inform operator which basic event is more important than the other. The famous basic events that cause major failures during the operation of NPPs include common cause failures (CCF), human error (HR), and man machine interface (Roberts et al., 1981; Cepin, 2002). To overcome these events some new techniques in Digital Instrumentation and Control (DI \& C) system has been introduced recently but according to some experts' opinion these digital methods increase chances of errors due to system own failure probabilities.

Hardware failure data and HR probability can also be deal with conventional PSA to a limited extent. But how to estimate HRs in qualitative manner and prioritize all consequences in case of accidents so that allocation of budget can be arranged in proper way are still important issues. The living probabilistic safety assessment (LPSA) and risk monitoring can be categorized as more advance terms as compare with PSA. In LPSA and risk monitoring, it is required to update PSA data by keeping in view prior probabilities (Zubair and Zhang, 2013; Zubair and Heo, 2013). These prior probabilities updated to posterior probabilities, which makes a guide line for decision makers and experts for their judgments. To make this decision making process more easy and accurate the present study uses analytic hierarchy process (AHP) and Bayesian network (BN) hybrid approach to increase reliability and safety.

\section{MATERIALS AND METHODS}

The methodology consists of AHP and BN combine method. The purpose of AHP is to develop a hierarchy and calculate prior probabilities. Then these prior probabilities have been used to calculate node probability table (NPT) in BN.

The AHP is a tool for solving multi-criteria decision problems. Analytic hierarchy process proposed by Saaty (1987) is very popular and has been applied in wide variety of areas including

- Planning

- Selecting a best alternative

- Resource allocation and resolving conflicts

Figure 1 shows that how AHP works for the selection of criteria to support decision makers. For simplicity, the reduction in criteria has been proposed, which leads to sufficient decrease in errors that appears in the selection of criteria.

The pair wise comparison has been done by using following four steps (Ishizaka and Labib, 2011)

- Structuring of the decision problem

- Making pair wise comparisons and obtaining the judgmental matrix 
- Computing local weights and consistency of comparisons

- Aggregation of local weights

To select suitable criteria by using only AHP is a difficult task but this difficulty can be resolve by using BBN. In fact, BBN provides a support to AHP in order to select criteria. During the selection of precursors there are many conditions and factors, which will be divided into two parts as represented in Figure 2. This figure explains the role of expert and precursors by considering internal

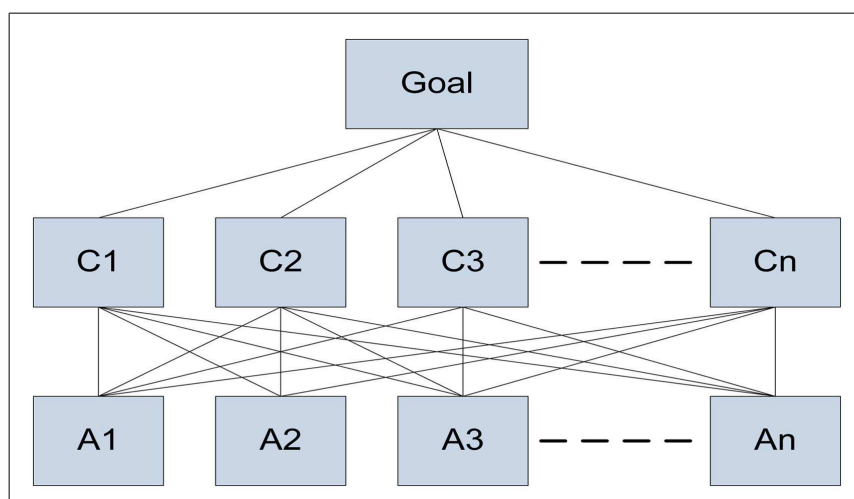

FIGURE 1 | Basic steps in AHP technique.
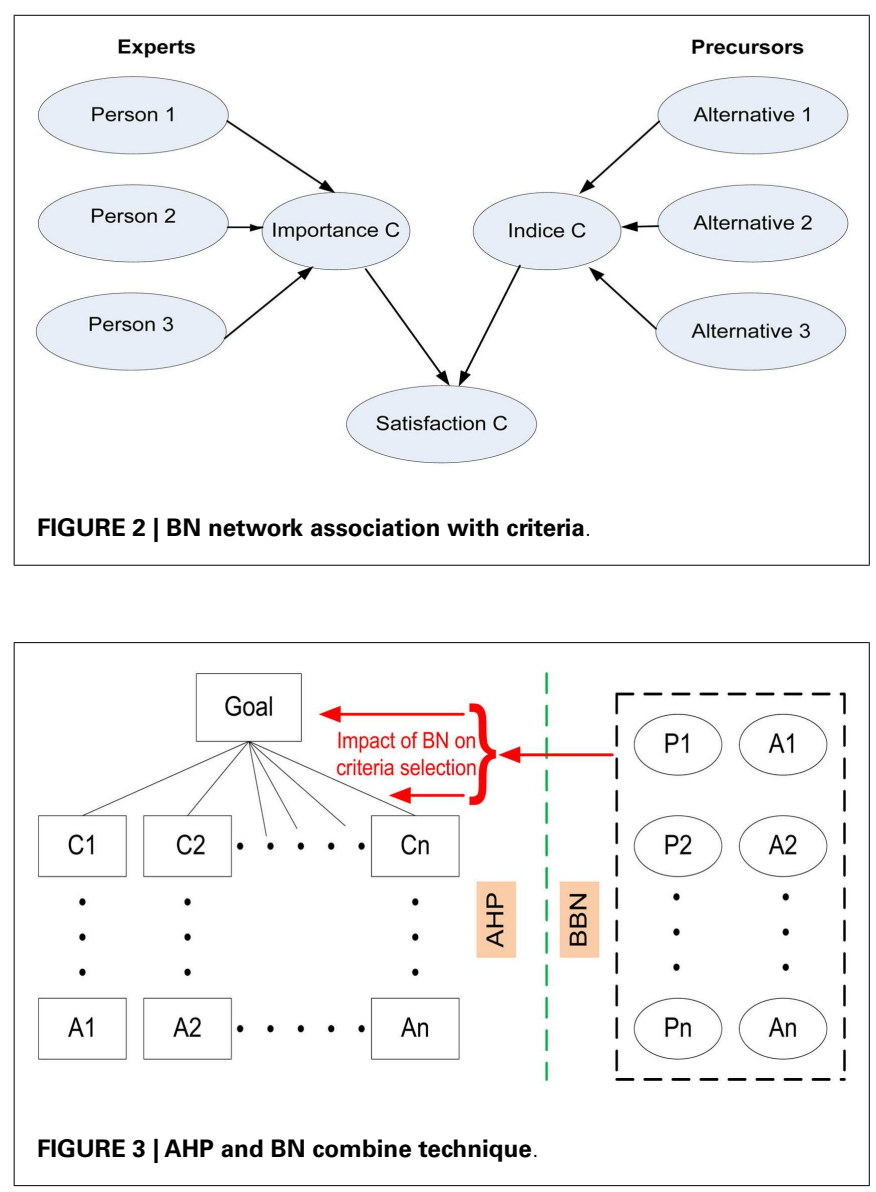

and external abilities. The following terms explain the role of $\mathrm{BN}$ in the selection of criteria.

Expert/Person: This is a person who will make selection. This person may belong to any region in the world having any kind of expertise.

Alternatives: These are the choices available. In our case, these are precursors that play a major role in causing any accident.

Index of criteria (indice C): It is a numerical function that affects all alternatives and represents quality of all alternatives.

Importance of criteria (Importance C): It represents the level of importance of criteria for experts (depends upon characteristics of experts).

Satisfaction of criteria (Satisfaction C): It denotes the level of satisfaction of experts to select precursors.

Once the characteristics of the person have been entered in the $\mathrm{BN}$, the importance is set for each criterion. After that, the value of the overall satisfaction is set to the highest value and propagation is done with inference. The first result is the posterior probabilities of the node of alternatives. The connection between three nodes,

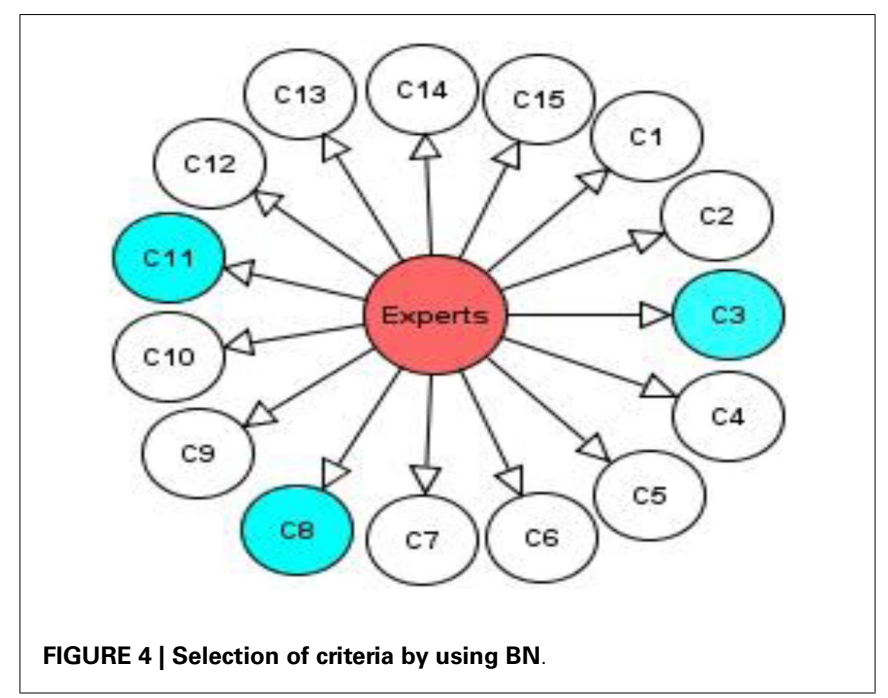

Table 1 | List of expected criteria.

\begin{tabular}{ll}
\hline Criteria & Description \\
\hline C1 & Availability of facilities (AF) \\
C2 & Defense in depth (DiD) \\
C3 & Operation (O) \\
C4 & Radiation protection (RP) \\
C5 & Quality management (OM) \\
C6 & Maintenance (M) \\
C7 & External events (EE) \\
C8 & Design (D) \\
C9 & Site characteristics (SC) \\
C10 & Common cause failures (CCF) \\
C11 & Safety (S) \\
C12 & Organizational aspects (OA) \\
C13 & Control room (CR) \\
C14 & Social aspects (SA) \\
C15 & Protection against disaster (PD)
\end{tabular}




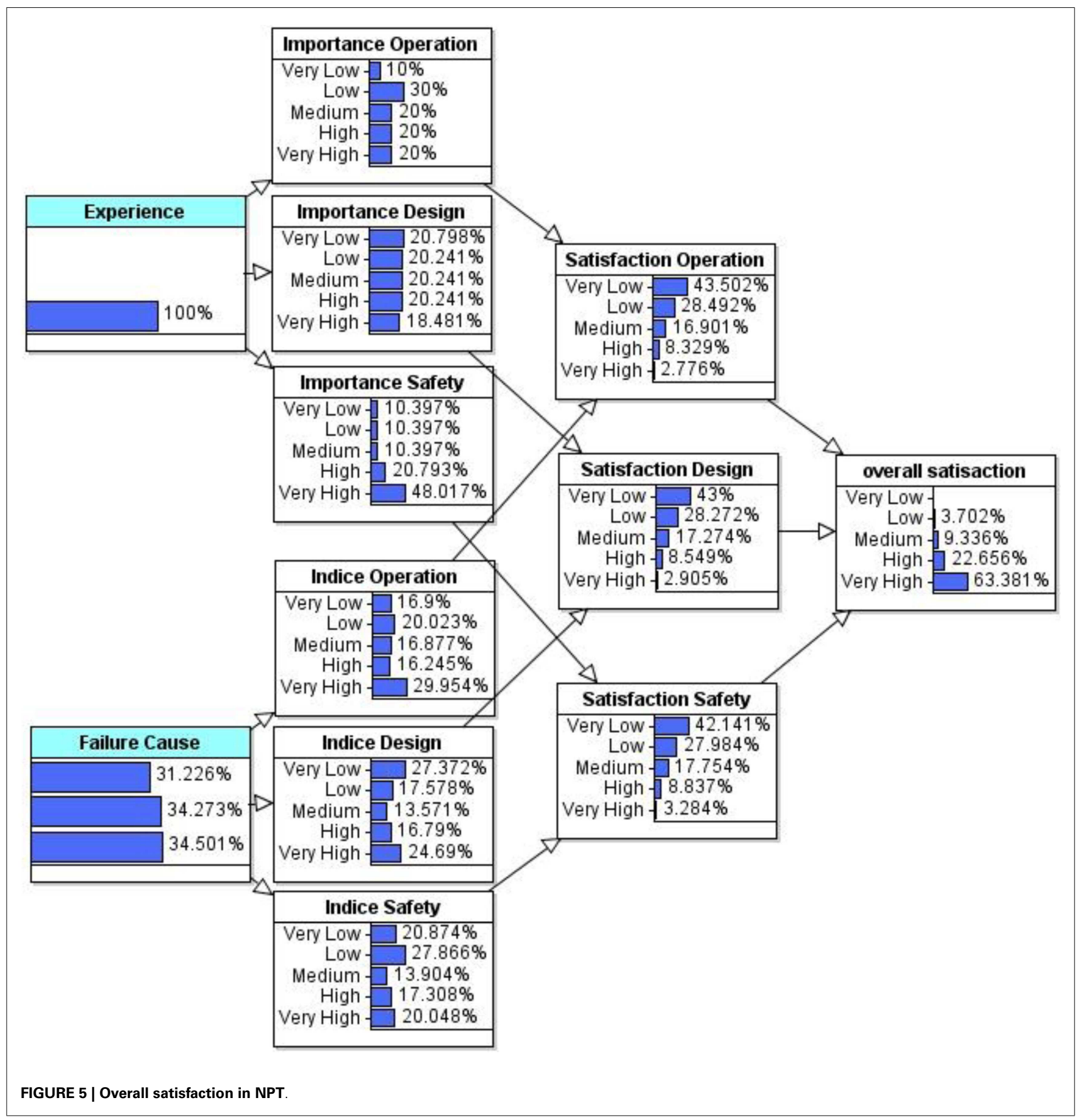

i.e., index, importance, and selection of criteria can be seen in Figure 2.

In $\mathrm{AHP}$ and $\mathrm{BN}$ combine method, the purpose of $\mathrm{BN}$ is to select most suitable criteria or precursors and provide this output into AHP as represented in Figure 3. On the basis of person's characteristics and the impact of all expected alternatives on criteria, $\mathrm{BN}$ choose a criterion that is satisfied by all aspects. These selected criteria pair wise compared in AHP and on the basis of weights of each pair AHP select best alternative.

\section{RESULTS AND DISCUSSION}

For instance in present research, the experts belong to different expertise, age, profession, region, and behavior analyze the accident in nuclear industry. Table 1 represents the selection of most important criteria from a given range of expected criteria.

Each criterion in Table $\mathbf{1}$ belongs to International Atomic Energy Agency (IAEA) safety standards (IAEA, 2000) safety standards. These standards have been considered in this study by 
keeping in view the safety management, technical requirements, plant design requirements, and safety objectives. It seems very difficult to analyze accident by keeping in view these 15 criteria but by using $\mathrm{BN}$ only top three criteria $(\mathrm{C} 3, \mathrm{C} 8, \mathrm{C} 11)$ have been selected by considering the expert's judgment as shown in Figure 4.

To understand the overall satisfaction of experts on these selected criteria the NPT has been selected such that it ranked satisfaction in five steps (very low, low, medium, high, and very high) as represented in Figure 5. The experts or judges having different years of experience need to set this NPT. In our case, experts have three type of experience $(10,20$, and 30 years). In Figure 5 only one scenario has been considered in which experts has 30 years of experience.

Now the next step is to use these criteria into AHP and decide best alternative that has most influence in causing nuclear accidents. Figure 6 represents AHP hierarchy structure having goal, criteria, subcriteria, and alternatives.

The subcriteria for plant operation divided into four classes as Human Factor (HF), Operator Training (OT), Dishonesty of Staff (DS), and Safety Culture (SC). In the same way, plant design is categorized into three types including Emergency Diesel Generator

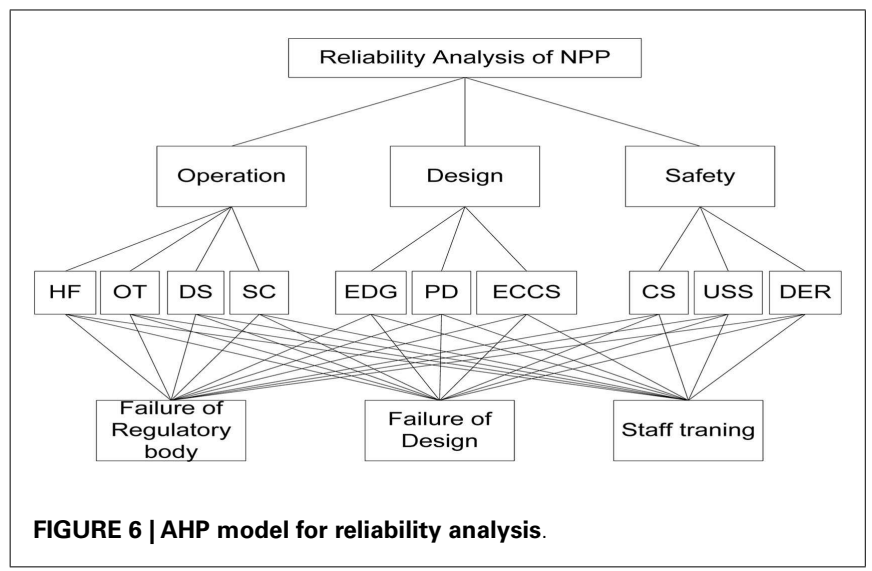

(EDG), Protection against Disaster (PD), and Emergency Core Cooling System (ECCS). The safety related issues divided into Component Survivability (CS), Unavailability of Safety System (USS), and Deficient Emergency Response (DER). Figure 7 represents that the failure of regulatory body has main contribution in context of plant design, operation, and safety as criteria.

By using AHP and keeping in view plant operation, design, and safety, the best cause of accident has been explored with the help of expert's judgments. On the basis of these judgments the total budget can be divided in such a way that NPP safety and regulatory become first priority, then management and design, respectively. So the best alternative to improve reliability of NPP is to improve regulatory body or in other words weakness in regulations decreases reliability as a result failure probability increases and causes accidents (Figure 8).

\section{CONCLUSION}

By using AHP, a methodology to increase NPP safety and reliability has been proposed. With the help of this technique the qualitative aspect of nuclear accident has been examined by considering

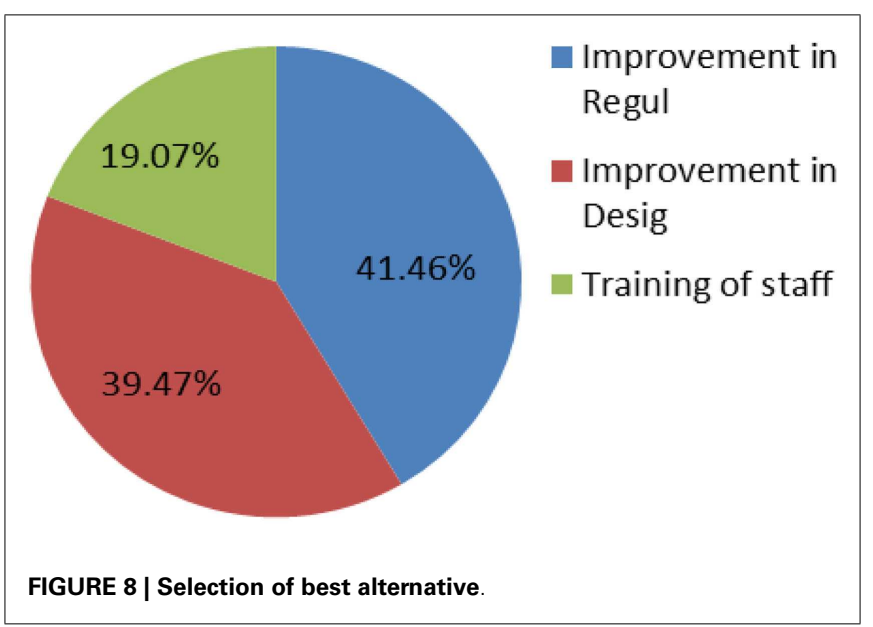

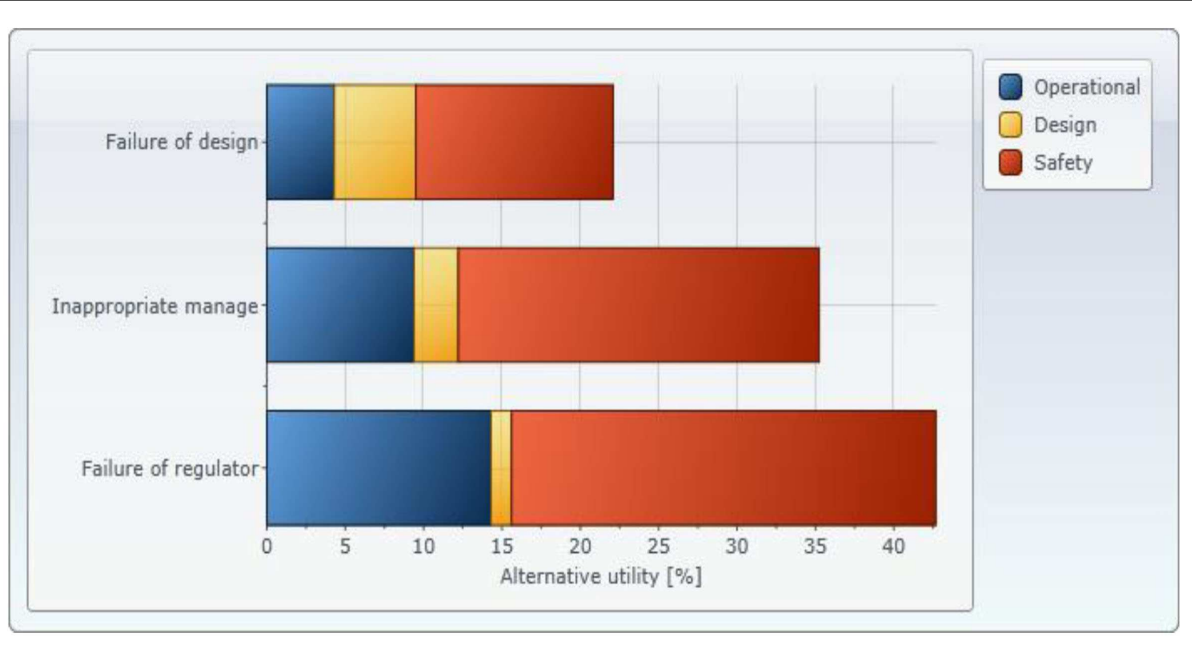

FIGURE 7 | Main cause of accident in context of criteria. 
experts judgment. The results showed that a lack of regulatory authorities was one of the main causes of accident. So, more budget allocation in this area would be helpful to reduce accidents and to improve nuclear safety. In this paper by using $\mathrm{BN}$, a method has been developed that initially uses 15 criteria and select most pertinent criteria (C3, C8, and C11). These selected criteria extracted by $\mathrm{BN}$ have been used in AHP. These outputs from BN provides to AHP in the form of weights of each criteria. On the basis of these weights AHP priorities all given data in the form of pair wise comparison and choose best alternative. So AHP and BN combine methodology as presented in this study provides decisions by keeping in view the quantitative and qualitative aspects of decision makers. The consistency ratio of weights provides a check system for the correctness of results obtained from AHP.

From this paper it is also clear that the decision depends on the characteristics of the decision maker that increases the quality of judgments as a result policy makers can easily decide for budget allocation in right direction. From this study, it is also clear that there is a need to make more attention and budget allocation to improve regulatory body as a result reliability of NPP will increase and accidents or failure probability will decrease.

\section{REFERENCES}

Ahmed, R., Koo, J., Jeong, Y., and Heo, G. (2011). Design of safety-critical systems using the complementarities of success and failure domains with a case study. Reliab Eng Syst Saf. 96, 201-209. doi:10.1016/j.ress.2010.09.008

Apostolakis, G., and Lemon, D. (2005). A screening methodology for the identification and ranking of infrastructure vulnerabilities due to terrorism. Risk Anal. 25, 361-376. doi:10.1111/j.1539-6924.2005.00595.x

Cepin, M. (2002). Optimization of safety equipment improves safety. Reliab Eng Syst Saf. 77, 71-80. doi:10.3171/2012.9.FOCUS12184

Haasl, D., Young, J., and Cramond, W. R. (1985). Probabilistic Risk Assessment Course Documentation, Vol. 4. Washington, DC: US NRC. NUREG/CR-4350.
IAEA. (2000). Safety Standards Series No. NSR-1, Safety of Nuclear Power Plants Design. Vienna: IAEA.

Ishizaka, A., and Labib, A. (2011). Selection of new production facilities with the group analytic hierarchy process ordering method. Expert Syst Appl. 38, 7317-7325. doi:10.1016/j.eswa.2010.12.004

NUREG-0492. (1981). Fault Tree Hand Book. Washington, DC: US Nuclear Regulatory Commission.

Roberts, N. H., Vesely, W. E., Haasl, D. F., and Goldberg, F. F. (1981). Fault Tree Hand-Book. Washington, DC: US NRC. NUREG-0492.

Saaty, T. L. (1987). Risk-Its priority and probability: the analytic hierarchy process. Risk Anal. 7, 159-172. doi:10.1111/j.1539-6924.1987.tb00980.x

Youngblood, R. (1998). Applying risk models to formulation of safety cases. Risk Anal. 18, 433-444. doi:10.1111/j.1539-6924.1998.tb00358.x

Zubair, M., and Heo, G. (2013). Advancement in living probabilistic safety assessment (LPSA) to increase safety of nuclear power plants. J Risk Reliab. 227, 534-539. doi:10.1177/1748006X13485192

Zubair, M., and Zhang, Z. (2013). Reliability data update method (RDUM) based on living PSA for emergency diesel generator of Daya Bay nuclear power plant. Saf Sci. 59, 72-77. doi:10.1016/j.ssci.2013.04.011

Conflict of Interest Statement: The author declares that the research was conducted in the absence of any commercial or financial relationships that could be construed as a potential conflict of interest.

Received: 19 September 2014; accepted: 03 November 2014; published online: 18 November 2014.

Citation: Zubair M (2014) A hybrid approach for reliability analysis based on analytic hierarchy process and Bayesian network. Front. Energy Res. 2:52. doi: 10.3389/fenrg.2014.00052

This article was submitted to Nuclear Energy, a section of the journal Frontiers in Energy Research.

Copyright (C) 2014 Zubair. This is an open-access article distributed under the terms of the Creative Commons Attribution License (CC BY). The use, distribution or reproduction in other forums is permitted, provided the original author(s) or licensor are credited and that the original publication in this journal is cited, in accordance with accepted academic practice. No use, distribution or reproduction is permitted which does not comply with these terms. 